\title{
COMPUTER SIMULATION OF THE HEAT TRANSFER IN A TOOL FOR REINFORCEMENT STEEL PRODUCTION
}

\author{
G. Evt. Georgiev*, V. Manolov, Y. Lukarski \\ AND AT. BAIKUSHEV \\ Institute of Metal Science, Equipment and Technologies \\ with Hydro- and Aerodynamics Centre "Akad. A. Balevski", \\ Bulgarian Academy of Sciences, \\ 67, Shipchenski Prohod Blvd, Sofia 1574, Bulgaria, \\ e-mails: g.georgiev@ims.bas.bg,v.manolov@ims.bas.bg, \\ lukarski@ims.bas.bg,nbaykushev@yahoo.com
}

A mathematical model describing the heat transfer processes in an instrument for cold drawing of reinforcement steel with round cross-section is performed. The model includes the system of nonlinear differential equations of heat conduction, heat transfer and the necessary set of boundary conditions for all details of the instrument. Some model solutions for different regimes of the used machine are obtained and presented. The comprehensive software package MAGMAsoft ${ }^{1}$ is used for this purpose. The influences of the geometry and intensity of the cooling on the temperature of the working surface of the nozzle are investigated and discussed. The obtained results are analysed and the main conclusions as a result of the made investigations are presented.

Keywords: mathematical modelling, temperature problem, heat exchange, extrusion.

\section{INTRODUCTION}

Currently, there are a wide variety of methods which form articles of metals and alloys. A great deal of them are the various casting methods of casting formation, such as low-pressure die-casting $[1,2]$, gas counter pressure casting $[3,4]$, centrifugal casting $[5,6,7]$ and methods forming new casting materials $[8,9]$. Together with the development of innovative technologies in these fields

\footnotetext{
${ }^{*}$ Corresponding author.

DOI: 10.7546/EngSci.LV.18.01.03

${ }^{1}$ MAGMAsoft is a trademark of MAGMA GmbH, Aachen, Germany.
} 
more progress is achieved by methods of mathematical modelling [10, 11], computer simulation [12] and optimization [13, 14] of technological solutions.

Another group of methods uses cold and hot plastic deformation to create articles of metals and alloys. One of the most widely used of them is the cold-drawing method of reinforcement iron.

The process of drawing of reinforcement steel through a nozzle is characterized by heat emission generated by two separate sources: one is due to the surface friction between the drawing tool and the material being drawn; the second is generated by the high rates of plastic deformation due to the reduction of the material cross-section diameter.

These two heat emissions lead to increased temperatures for both the drawn material and the nozzle, which reflect negatively on the nozzle strength, durability and ultimately its lifetime. Therefore, the investigation of the temperature regime of the assignment tool is very important for optimization of the technological process and for increasing its productivity and efficiency.

This article presents the results of mathematical modelling of processes of heat transfer in the system of parts involved in the drawing block. The temperature problem is solved for different regimes of the instrument.

\section{BACKGROUND OF THE PROBLEM}

The main components of the drawing tool are tungsten carbide nozzle and bushing made from steel 45X. They are joined by a shrink fit. Geometrical dimensions of the nozzle are presented in Fig. 1 and a cross-section of both components of the instrument is pointed out in Fig. 2.

The boundary condition for the temperature problem on the working surface of the nozzle is determined following the approach proposed in [15]. As discussed there, the force needed to draw reinforcing steel with circular section through a conical nozzle can be defined by the formula:

$$
F=\frac{1}{2}\left(R_{02}^{0}+R_{02}\right)\left(S_{0}-S\right)(1+f c t g \alpha),
$$

where $R_{02}^{0}$ is the tensile strength of the reinforcing steel before draw; $R_{02}-$ tensile strength of the reinforcing steel after draw; $S_{0}$ - cross-section of the reinforcing steel before draw; $S$ - cross-section of the reinforcing steel after draw; $f$ - friction coefficient between reinforcing steel and working surface of the nozzle; $\alpha$ - the angle between the generatrix of the conical surface of the nozzle and axis of rotation symmetry (Fig. 1).

(c) Инженерни науки, год. LV, 2018, № 1 


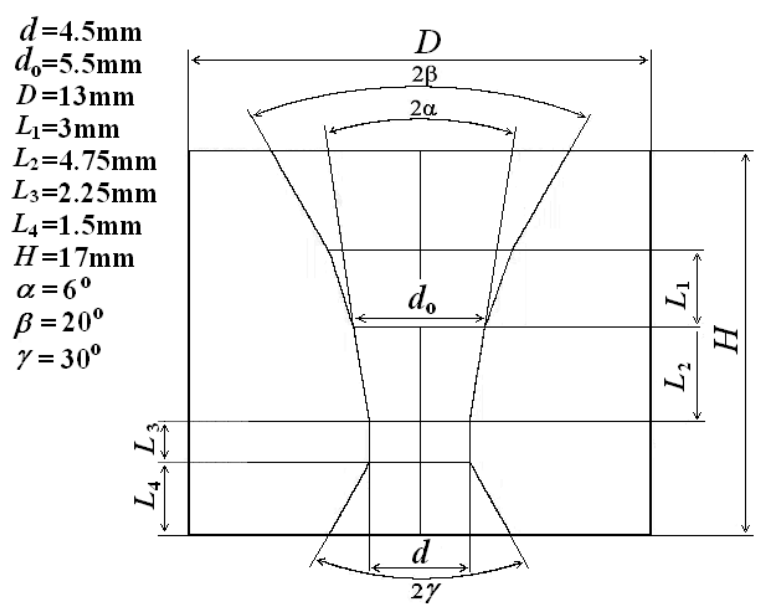

Fig. 1. Drawing of the nozzle

Taking into account equation (1) the power required for withdrawal should be determined by the formula:

$$
P=F v,
$$

where $v$ is the draw velocity. Part of this power is separated in the form of heat due to internal friction in the material as a result of its plastic deformation. According to the research [16], this heat remains almost entirely in reinforcing steel. Another part of the power is consumed to overcome the friction between the surface of the reinforcing steel and the working surface of the nozzle. It is also converted to heat. One part of it is given to the instrument and the other one is taken away from reinforcing steel.

In [15] the following estimation of the magnitude of the heat flux entering in the nozzle through its working surface is obtained:

$$
q_{n}=\frac{500 \delta^{2}+5 \sqrt{d v}}{3 d v+5 \sqrt{d v}} \frac{P_{f}}{S_{W}}
$$

where

$$
\begin{gathered}
\delta=\frac{S_{0}-S}{S}, \\
P_{f}=1.2(0.77-\sqrt{\delta}) P,
\end{gathered}
$$

$S_{W}$ is the area of the working surface of the nozzle in $\mathrm{mm}^{2} ; v$ - the draw velocity in $\mathrm{m} / \mathrm{min}$; $d$ - the final diameter of the reinforcing steel in $\mathrm{mm}$ (pointed out in Fig. 1).

(c) Инженерни науки, год. LV, 2018, № 1 


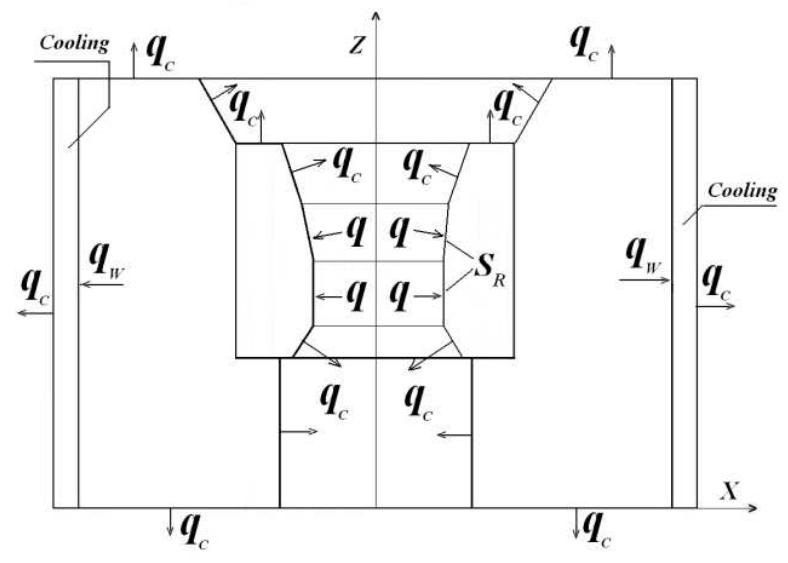

Fig. 2. Cross-section of the instrument

Constants in formula (3) have such kind of dimensions that lead to dimensionless value for the first fraction there.

Technological parameters of the used machine for draw provide stable process by $v=180 \mathrm{~m} / \mathrm{min}$. Consumed standard lubricant provides the following coefficient of friction: $f=0.06$. Using the values above and also the specific geometrical dimensions aligned in Fig. 1, after substitution into equations from (1) to (5), for the magnitude of the heat flux on the nozzle working surface the following value is obtained:

$$
q=3 \cdot 68 \cdot 10^{6}, \quad \mathrm{w} / \mathrm{m}^{2} .
$$

This value is used as a boundary condition of the temperature problem of the instrument. The standard assumption that the heat flux direction is perpendicular to the work surface is accepted.

The main components of the process of heat transfer in the tool are presented in Fig. 2. The free surfaces of the instrument are cooled by air convection. The intensity of this heat flux is denoted by $q_{C}$. Cooling with intensity of the heat flux $q_{W}$ is supposed to act on ambient surface of the steel bushing.

After a sufficient period of time, temperature field in the tool reaches steady state and the temperature on $S_{R}$ occupies its important operating value, respectively.

The present work aims to obtain steady-state of the temperature field and the operating temperature on $S_{R}$.

(c) Инженерни науки, год. LV, 2018, № 1 
For this purpose the 3D heat transfer equation:

$$
\frac{\partial\left(c_{p} \rho T\right)}{\partial t}=\operatorname{div}(\lambda \operatorname{grad}(T))
$$

is solved for all spatial areas of the instrument.

The problem is solved under the following initial and boundary conditions:

1. Boundary condition of heat flow that heats the nozzle through its working surface $S_{R}$ :

$$
q=\lambda_{1} \frac{\partial T}{\partial n}
$$

The intensity of heat flux and the derivative of the temperature field are supposed as normal to that surface.

2. Boundary condition for free convection on surfaces which are in contact with ambient air:

$$
\lambda \frac{\partial T}{\partial n}=q_{C}=\alpha_{C}\left(T_{\Pi}-T_{E}\right),
$$

where $q_{C}$ is the heat flow in these areas (Fig. 2); $\alpha_{C}$ - the heat transfer coefficient of convection; $T_{\Pi}$ - the temperature at the corresponding points of the free surfaces; $T_{E}$ - the air temperature of the environment.

3. Boundary condition in the area of thermal contact in the shrink fit between the two bodies:

$$
-\lambda_{1} \frac{\partial T}{\partial n}=\alpha_{p s}\left(T_{P 1}-T_{P 2}\right)=-\lambda_{2} \frac{\partial T}{\partial n},
$$

where $T_{P 1}, T_{P 2}$ are the temperatures of the two surfaces in contact; $\lambda_{1}, \lambda_{2}-$ the corresponding coefficients of thermal conductivity of the two bodies; $\alpha_{p s}$ - the heat transfer coefficient in the shrink fit.

In many cases of practice the instrument is placed in a cooler. For these cases the following boundary condition should be added:

4. Boundary condition of the contact surface between the steel bushing and the cooler:

$$
\lambda \frac{\partial T}{\partial n}=q_{W}=\alpha_{\text {cooling }}\left(T_{2}-T_{\text {cooling }}\right),
$$

where $q_{W}$ is the intensity of heat flow given from steel sleeve to the cooling; $\alpha_{\text {cooling }}$ - the heat transfer coefficient on the bushing-cooler boundary; $T_{2}-$ the surface temperature of the steel sleeve in contact with the surface of the cooler; $T_{\text {cooling }}$ - the temperature of the cooling fluid.

Some of the necessary thermal parameters are taken from the literature [16-18] and others from the database of the software package MAGMAsoft. Most of these parameters are nonlinear functions of temperature. The data for

(C) Инженерни науки, год. LV, 2018, № 1 
the heat transfer coefficient $\alpha_{p s}$ is obtained by averaging the results published in [19] and [20]. Database of MAGMAsoft is used for the determination of $\alpha_{\text {cooling. }}$

\section{NUMERICAL SOLUTIONS OF THE MODEL}

The mathematical model mentioned in previous section (equations 7-11) is used to calculate the temperature field of the instrument (named "calibration block" below) shown in Fig. 3. It includes:

- nozzle (Part 1);

- steel bushing (Part 2);

- system for mounting and cooling (Part 3) the whole tool into the drawing machine.

Temperature fields are obtained for all parts of the tool in different modes and different types of coolers. The results are used for identification and analysing the thermal load of the tool at different modes of drawing and different cooling schemes. The results obtained for three different cases of the cooling are given below.

\section{A) Case 1}

This case includes a water cooler in the form of a hollow cylinder (ring) covering the bushing (Part 2, from Fig. 3). Geometric configuration used in this option is implemented in Fig. 3 schematically in a cross-section. For the heat flux through the surface of the nozzle the value from (6) is taken. Some control points at different radii from the axis of symmetry are chosen. Their values for considered three cases are given in Table 1.

TABLE 1

\begin{tabular}{|c|c|c|c|c|c|c|c|}
\hline \multicolumn{2}{|c|}{ No } & $T_{1}$ & $T_{2}$ & $T_{3}$ & $T_{4}$ & $T_{5}$ & $T_{6}$ \\
\hline \multicolumn{2}{|c|}{$\mathrm{R}[\mathrm{mm}]$} & 3.2 & 4.2 & 5.2 & 6.6 & 10.02 & 10.02 \\
\hline Case 1 & \multirow{3}{*}{$\mathrm{T}\left[{ }^{\circ} \mathrm{C}\right]$} & 201.3 & 157.7 & 138.5 & 119.3 & 95.41 & 61.96 \\
\hline Case 2 & & 219.6 & 175.5 & 156.4 & 137.5 & 113.9 & 73.0 \\
\hline Case 3 & & 382.4 & 341.3 & 323.3 & 305.2 & 282.6 & 244.1 \\
\hline \multicolumn{2}{|c|}{ No } & $T_{7}$ & $T_{8}$ & $T_{9}$ & $T_{10}$ & $T_{11}$ & \\
\hline \multicolumn{2}{|c|}{$\mathrm{R}[\mathrm{mm}]$} & 13.0 & 14.8 & 18.0 & 21.0 & 25.0 & \\
\hline Case 1 & \multirow{3}{*}{$\mathrm{T}\left[{ }^{\circ} \mathrm{C}\right]$} & 45.23 & 42.18 & 36.19 & 33.23 & 29.81 & \\
\hline Case 2 & & 65.2 & 60.6 & 55.9 & 52.5 & 49.3 & \\
\hline Case 3 & & 236.2 & 231.8 & 228.1 & 224.5 & 221.8 & \\
\hline
\end{tabular}

(c) Инженерни науки, год. LV, 2018, № 1 
The flow rate of cooling water is kept at $1 \mathrm{~m}^{3} / \mathrm{h}$ and the temperature of incoming water is taken as $25^{\circ} \mathrm{C}$.

Under the conditions described above, the temperature problem formulated in Section 2 is solved using MAGMAsoft software. The steady state is reached in a relatively short time - less than 1 min. Figure 4 presents the obtained steady state of the temperature field in the Part 1.

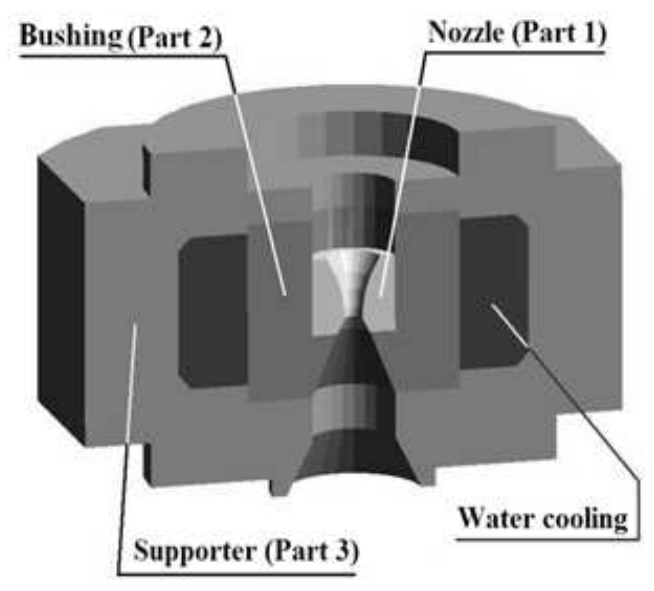

Fig. 3. The parts of the calibration block

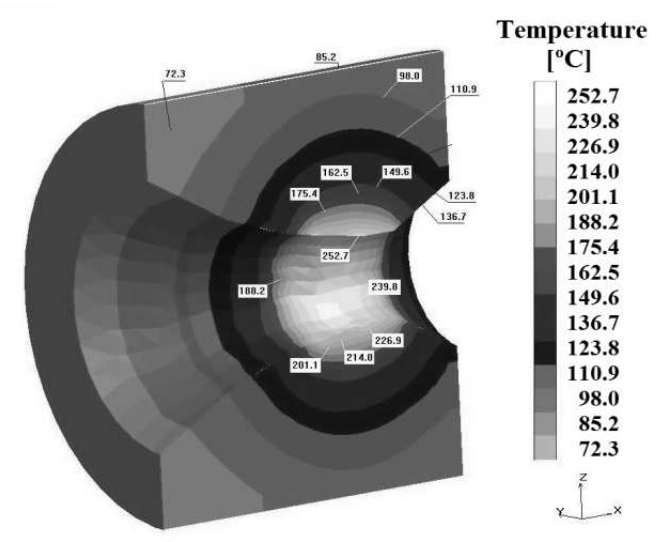

Fig. 4. Steady state temperature field of the nozzle

The grey scale encoding is used for this purpose. Figures 5 and 6 show the related temperature field of the bushing (Part 2) and of the Part 3 (Fig. 3 ). Some badges with digital values of the temperatures in different zones are used for greater clarity.

(c) Инженерни науки, год. LV, 2018, № 1 


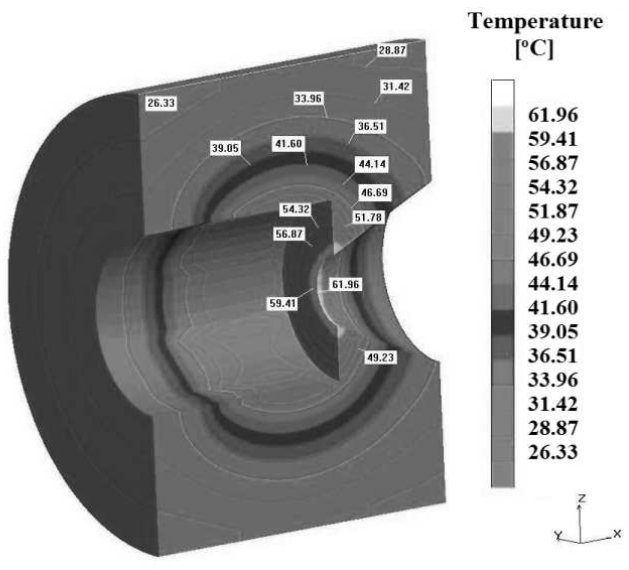

Fig. 5. Temperature field of Part 2

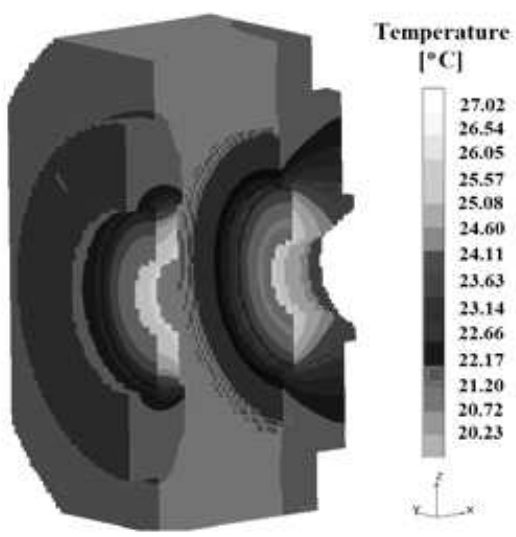

Fig. 6. Temperature field of Part 3

Figure 7 shows the steady state temperature distribution in the radial direction (temperatures at control points from Table 1, Case 1). The typical jump of the temperature behaviour in the contact zone between Par 1 and Part 2 is observed. The calculated temperature of the surface $S_{R}$ is approximately $253{ }^{\circ} \mathrm{C}$ (see the maximum temperature in Fig. 4).

\section{B) Case 2}

This case differs from the Case 1 with the geometry of the water cooling. It is constructed here as cylindrical channels pointed out in Fig. 8. All other conditions are the same as in Case 1 . The temperature problem is also solved

(c) Инженерни науки, год. LV, 2018, № 1 


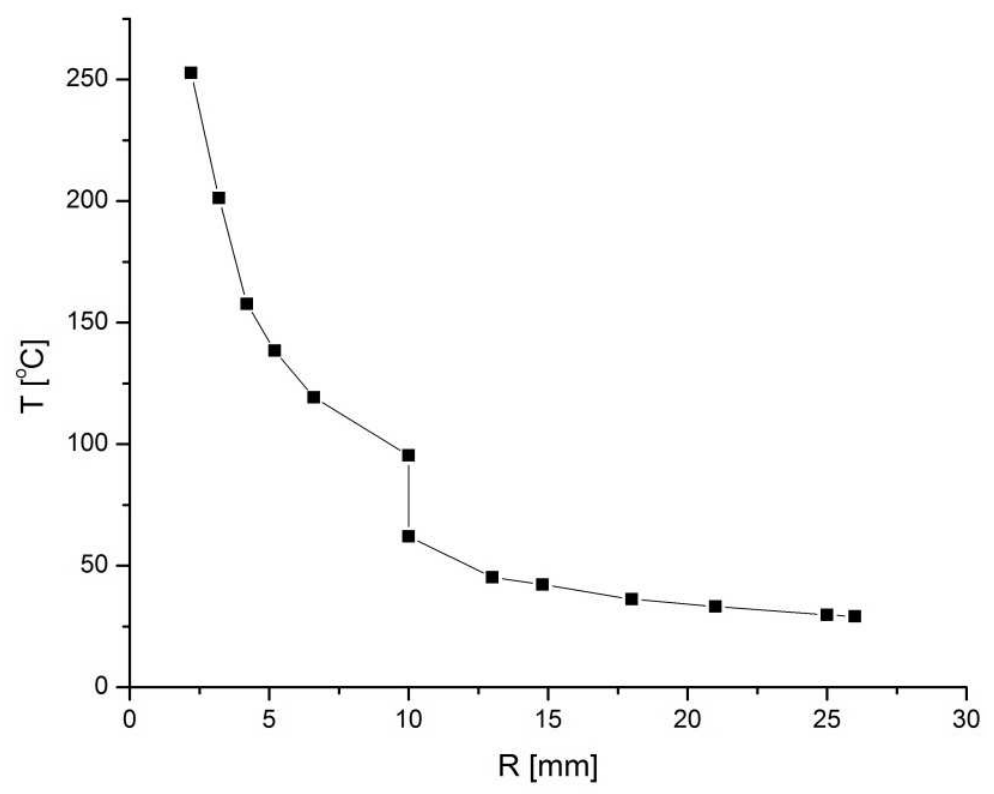

Fig. 7. Temperature in control points

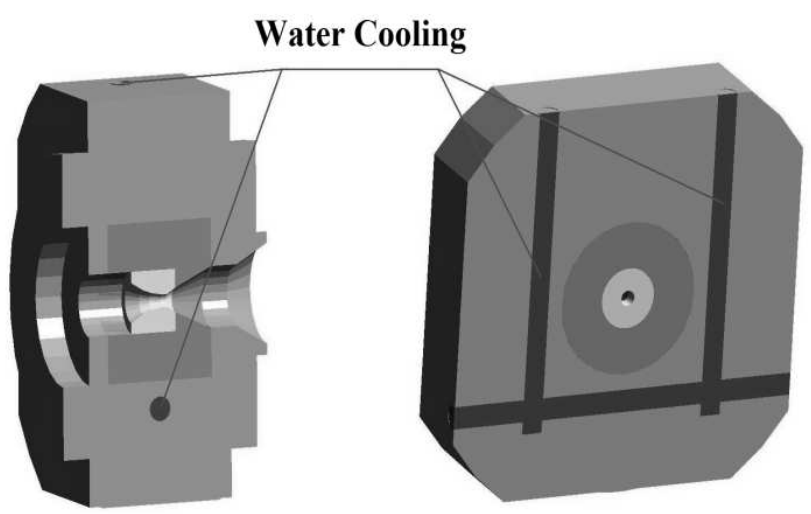

Fig. 8. Cooling in Case 2

with MAGMAsoft for Part 1, Part 2 and Part 3. The obtained temperature field of Part 1 is shown in Fig. 9. The comparison whit this one from Fig. 4 shows that the temperature on the work surface at the Case 2 is $16^{\circ} \mathrm{C}$ higher than the respective temperature in Case 1 . This result suggests that the cylin-

(c) Инженерни науки, год. LV, 2018, № 1 
drical cooling from Fig. 3 is more efficient than the version with cylindrical channels (Fig. 8). However, when comparing the time to reach the steady state, there is no significant difference between the two considered cases.

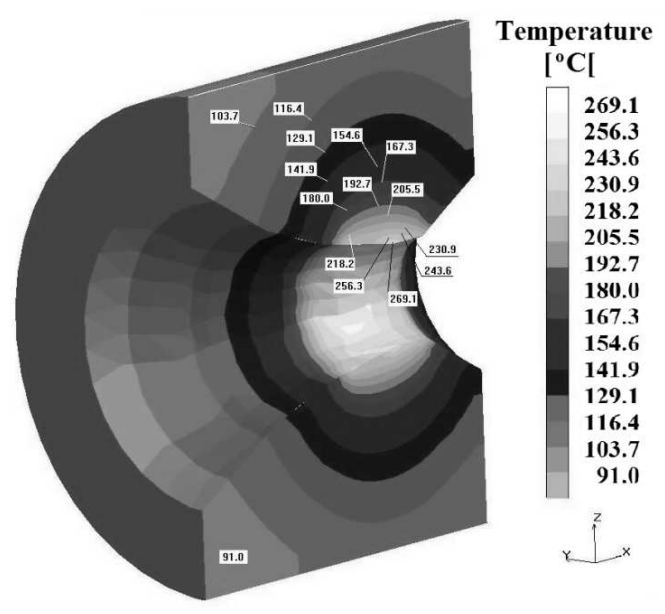

Fig. 9. Temperature field of Part 1. Case 2

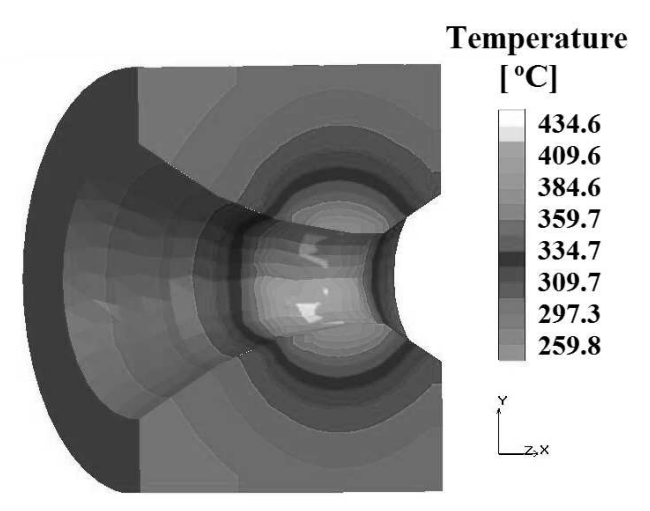

Fig. 10. Temperature field of Part 1. Case 3

\section{C) Case 3}

This case studies the configuration when the same instrument works without cooling. Only the free air convection to the environment and heat transfer through mechanical contacts are taking into account. The temperature problem is also solved with MAGMAsoft for Part 1, Part 2 and Part 3. The obtained solution for most important temperature field of Part 1 in this case is shown in Fig. 10. It is seen that the temperature on the work surface of

(c) Инженерни науки, год. LV, 2018, № 140 (c) Engineering Sciences, LV, 2018, No. 1 
the nozzle rises to approximately $435{ }^{\circ} \mathrm{C}$. This temperature is higher than the operating temperature of Case 1 (Fig. 4) with more than $180^{\circ} \mathrm{C}$ and approximately with $166^{\circ} \mathrm{C}$ higher than the one in Case 2 (Fig. 9). The steady state in this case is reached for approximately 3 hours, while water-cooled cases require only approximately 30 seconds.

\section{CONCLUSION}

Based on the results above the following conclusions could be made:

- Water cooling reduces significantly the operating temperature of the drawing tool. Therefore it increases the durability and lifetime of the instrument;

- Additional water cooling provides faster transition from non-stationary to stationary temperature regime. This fact is important for maintaining constant conditions throughout the whole production process;

- The presented two water cooling designs provide similar operating temperatures. Therefore the choice could be made by other reasons (e.g. structural or functional);

- The use of additional water cooling is advisable and even imperative for high performance regimes with high speeds of drawing (see the comparison between the results of Case 1-2 and Case 3).

\section{ACKNOWLEDGEMENTS}

This work is developed with the financial support of the "National Innovative Fund" by the contract No 4ИФ-02-80/07.03.2008.

\section{REFERENCES}

[1] J. J. Sobczak, L. Drenchev and R. Asthana, Effect of Pressure on Solidification of Metallic Materials, International Journal of Cast Metals Research (2012) 25 1-14.

[2] A. Maneva, S. Stanev and M. Georgiev, Investigation of the Possibilities of Applying Vacuum in the Mould Cavity by Low Pressure Die Casting of Aluminium Alloys, in: Sci. Proceedings of XXIX Int. Conf. "Defectoscopy'14", June 2014, Year XXII, 1(150), pp. 158-163, ISSN:1310-3946 (in Bulgarian).

[3] A. Maneva and S. Bushev, Technological design - Counter Pressure Die Casting, in: Proceedings of $5^{\text {th }}$ National Conf. with Int. participation, "Material Science, Hydro- and Aerodynamics and National Security'2015", Sofia, October 22-23, 2015, pp. 184-185, ISSN 1313-8308 (in Bulgarian).

(c) Инженерни науки, год. LV, 2018, № 1

41

(C) Engineering Sciences, LV, 2018, No. 1 
[4] L. Stanev and St. Bushev, Solidification at High Pressure, in: Proceedings of XXIV Int. Sci. and Technical Conf. "FOUNDRY 2017", Pleven, Bulgaria, April 5-7, 2017, Year I, pp. 44-46, ISSN: 2535-017x (print), ISSN 2535-0188 (online).

[5] I. Georgiev, S. Bushev, I. Penkov and S. Stanev, Centrifugal Casting of Cast Iron Billets for Metallic Conductors, in: Proceedings of $5^{\text {th }}$ National Conf. with Int. Participation, "Material Science, Hydro- and Aerodynamics and National Security'2015", Sofia, October 22-23, 2015, pp. 108-112, ISSN 13138308 (in Bulgarian).

[6] I. Georgiev and M. Bushev, Centrifugal Casting with Vertical Axis of Bimetal Castings for Rollers in the Ceramic Industry, in: Sci. Proceedings of XXX Int. Conf. "Defectoscopy'15", June 2015, Year XXIII, 2(165), pp. 181-184, ISSN: 13103946 (in Bulgarian).

[7] I. Georgiev, S. Stanev and S. Bushev, Centrifugal Casting and Mechanical Testing of Heat-resistant Alloys, in: Sci. Proceedings of XXIX Int. Conf. "Defectoscopy'14", June 2014, Year XXII, 1(150), pp. 114-117, ISSN: 1310-3946 (in Bulgarian).

[8] L. Stanev, M. Kolev and L. Drenchev, Application of Space Holders for Obtaining of metallic Porous Materials. Part I: Production Methods, Journal of Materials Science and Technology (2015) 23 (4) 303-344, ISSN: 0861-9786.

[9] L. Stanev, M. Kolev, B. Drenchev and L. Drenchev, Application of Space Holders for Obtaining of Metallic Porous Materials. Part III: Structure and Properties of Titanium and Titanium Alloy Foams. A Review, Journal of Materials Science and Technology (2016) 24 (2) 106-123, ISSN: 0861-9786.

[10] A. Maneva And S. Bushev, Measurement of Foundry Structures Mathematics, Int. Sci. J. "Industry 4.0", Scientific Technical Union of Mechanical Engineering, Bulgaria, (2017) II (5) 224-227, ISSN: 2543-8582 (Print), 2534997X (Web).

[11] V. Valkov, I. Georgiev and S. Bushev, Choice of Models to Describe the structure of Austenitic Alloy Formed by Centrifugal Casting, in: Sci. Proceedings of XXXI Int. Conf. "Defectoscopy'16", June 2016, Year XXIX, 1(187), pp. 229 232, ISSN: 1310-3946 (in Bulgarian).

[12] H. Skulev, B. Drenchev, T. Mechkarova and L. Drenchev, Kinetics of Surface Layers Growth during Gas Nitriding of Ti-6Al-4V by Indirect Plasma Torch, J. Material Science and Technology (2014) 22 (2) 57-75, ISSN: 0861-9786.

[13] G. Evt. Georgiev, N. Tonchev, I. Georgiev, P. Petrov and V. Videv, Computer Optimization of a Technology for Forming a Steel Casting, Engineering Sciences (2015) LII (2) 73-80, ISSN: 1312-5702.

[14] S. Bushev, A. Maneva And I. Georgiev, Possibility to Control the Phase Transformation during Casting Formation, in: Sci. Proceedings of XXIX Int. Conf. "Defectoscopy'14", June 2014, Year XXII, 1(150), pp. 108-109, ISSN: 13103946 (in Bulgarian).

(c) Инженерни науки, год. LV, 2018, № 1 
[15] I. A. Uhvets, Drawing Production, Part I, Metallurgia (1965) (in Russian).

[16] R. B. Krasilchikov, Heating at Cold Steel Drawing of a Strand, Metalurgizdat, Moscow (1962) (in Russian).

[17] M. A. Mineev And I. M. Mineeva, Fundamentals of Heat Transfer, Energia, Moscow (1977) (in Russian).

[18] Handbook Heat Conduction of Solids (Ed. A. S. Ohotina), Energoatomizdat, Moscow (1984).

[19] V. N. Karnozhitskir, Contact Heat Change during a Aasting, Naukova Dumka, Kiev (1978) (in Russian).

[20] U. P. Slikov, E. A. Ganin and S. N. Tsarevskil, Contact Thermal Resistance, Energia, Moscow (1977) (in Russian).

\title{
КОМПЮТЪРНА СИМУЛАЦИЯ НА ТОПЛОПРЕНОСА В ИНСТРУМЕНТ ЗА ПРОИЗВОДСТВО НА АРМИРОВЪЧНА СТОМАНА
}

\author{
Г. Евт. ГЕОРГИЕВ*, В. МАНОЛОВ, Яв. ЛУКАРСКИ \\ И АТ. БАЙКУШЕВ
}

Институт по металознание, сборбжения и технологии

с Центор по хидро- и аеродинамика „Акад. Ангел Балевски“, Българска академия на науките, бул. Шипченски проход № 67, 1574 София, Бглгария, e-mails:g.georgiev@ims.bas.bg,v.manolov@ims.bas.bg, lukarski@ims.bas.bg,nbaykushev@yahoo.com

Резюме. Представен е математичен модел, описващ процесите на топлопренос в инструмент за студено изтегляне на армировъчна стомана с кръгло сечение. Моделът включва система от нелинейни диференциални уравнения за топлопроводност, топлопренос и необходимия набор от гранични условия за всички детайли на инструмента. Получени и представени са моделни решения за различните режими на използваната машина. За тази цел е използван притежаващият висока степен на общност софтуерен пакет MAGMAsoft. Изследвани и дискутирани са влиянията на геометрията и интензивността на охлаждането върху температурата на работната повърхност на дюзата. Получените резултати са анализирани, като

(c) Инженерни науки, год. LV, 2018, № 1 
са представени основните заключения в резултат на направените изследвания.

Ключови думи: математическо моделиране, температурна задача, студено изтегляне.

Received February 07, 2018 